\title{
Tolerance Design of Curing Process of Electrically Conductive Adhesives
}

\author{
Seba Barto, Pavel Mach \\ Czech Technical University in Prague, Faculty of Electrical Engineering, Department of Electrotechnology \\ Prague, Czech Republic \\ mach@fel.cvut.cz
}

\begin{abstract}
The work was directed at examination of influence of tolerances of curing process parameters on the tolerances of the resistance and nonlinearity of a current $v$ s. voltage characteristic of adhesive joints. The joints were formed of electrically conductive adhesive with isotropic electrical conductivity of an epoxy type filled with silver flakes. Mathematical models for the resistance and nonlinearity were found using factorial experiments of the type $2^{2}$. Tolerance design was carried out for these models. It was found that tolerances of the resistance and nonlinearity depend more on the time of curing than on the curing temperature.
\end{abstract}

\section{INTRODUCTION}

Electrically conductive adhesives play growing role in electronics assembly. It is not possible to substitute them by soldering if a mounted component is temperature sensitive and the soldering temperature would destroy it.

It is not possible to substitute them if some finepitch packages are mounted, especially when a gap between leads is $0,3 \mathrm{~mm}$, because the use of soldering causes higher number of bridges between neighbor leads.

Quality of adhesive joining is strongly influenced by curing. The curing temperature of adhesives is 120 to $150{ }^{\circ} \mathrm{C}$ usually. The curing time is between 10 to 30 minutes. Curing causes hardening of adhesive and decrease of its volume.

The volume decrease plays significant role in quality of contacts among filler particles. The better is contact quality, the lower is the contact resistance and the lower is the joint resistance. Nonlinearity of the joint current vs. voltage characteristic and noise decrease too. Total contact quality increases.

The curing of adhesive joints is carried out in a tunnel oven usually. The ovens are heated either with resistance tube radiators or infrared emitters. The temperature profile inside oven is controlled electronically.

With respect to the fact that such an oven consists of 6 to 10 individually controlled sections, it is not easy to set up a requested temperature profile.

The control of sections is carried out with limited accuracy. Therefore the temperature profile is also set up with limited accuracy. With respect to the significance of the temperature processing of adhesives, which influence electrical as well as mechanical properties of adhesive joints, the tolerance of the oven temperature profile will influence properties of the joints. That is the reason why it is necessary to carry out the tolerance design of a process of adhesive curing.

\section{THEORETICAL BACKGROUND}

The tolerance design is defined as "The total amount by which a given dimension may vary or the difference between the limits" [1]. Tolerances occur in every stadium of a technological process. It is necessary to stress that every technological process and every technological operation are connected with some tolerances.

As for the process of adhesive joining, there are many sources of tolerances here. Different charges of 
the same adhesive can differ in viscosity, according to their storage time, processes of adhesive dispensing, screen or stencil printing will differ from the point of view of accuracy of amount dispersed or printed adhesive on a pad, tolerances of the temperature of adhesive curing will influence the hardening of adhesive and its shrinkage. Especially shrinkage can influence quality of contacts among conductive particles of adhesive and quality of conductive net inside it.

Tolerances joined with properties of adhesive, e.g. its viscosity, and their influence on adhesive joint properties are tested by adhesive producers. Influence of tolerances joined with technology of application of adhesive on the properties of adhesive joints is tested by printing machines producers. The goal of the work is to analyze influence of the curing temperature tolerance on electrical properties of the adhesive joints - the resistance and nonlinearity of the current vs. voltage characteristic.

Tolerance design is based on a study of a mathematical model [2]. The model is calculated using a method of factorial experiments of the type $2^{2}$. First the significance of influence of basis curing factors, the time and the temperature, on the output parameters - the resistance and nonlinearity of adhesive joints - is examined and tested and then significance of influence of interaction temperaturetime is examined and tested [3], [4]. Following step of calculation is transformation of technological factors in new variables and selection of a proper model equation [5]. The linear models are chosen for both output parameters of the curing process.

Transformation of technological factors in new variables is carried out to simplify the solution of normal equations.

$$
X_{1}=\frac{2}{A_{2}-A_{1}} \cdot\left(A-\frac{A_{1}+A_{2}}{2}\right)
$$

Where $X_{1} \ldots$ transformed technological factor $A, A_{1}$ ... lower specification limit of the factor $A, A_{2} \ldots$ upper specification limit of the factor $A$.

The value of transformed variables is -1 for minimum value of the technological factor and +1 for its maximum value. The method of least squares is used for calculation of coefficients of the mathematical model. After calculation of the model its accuracy is statistically tested. When the result is that the model is accepted for the work, theory of tolerance is applied. The detail process of calculation of the mathematical model is presented in the article Influence of Curing Parameters on Quality of Electrically Conductive Joints, which is a part of this proceeding.

Tolerance design is based on theory of tolerances [6] focused on two problems:

- To find tolerance of an output parameter of a process in surroundings of some working point if tolerances of input parameters of this process are known.

- To find tolerances of input parameters of a process if tolerance of an output parameter are known.

The second task is a mathematically incorrect task, because without some additional conditions it is possible to find unlimited number of solutions.

Absolute error (tolerance) of an output parameter, when the output parameter depends on one input parameter only, is calculated using a formula:

$\Delta_{y}=\Delta_{x} \cdot\left(\left|\frac{d y(x)}{d x}\right|\right)_{x 0}$

Here $\Delta_{y} \ldots$ absolute value of the output parameter $y, \Delta_{x}$ ... absolute value of the input parameter $\mathrm{x}, \mathrm{y}(\mathrm{x}) \ldots$ mathematical model, $X_{0} \ldots$ point, where tolerance is examined.

Absolute error (tolerance) of the output parameter, when the output parameter depends on more input parameters, is calculated using a formula:

$$
\begin{aligned}
& \Delta_{y}=\left(\left|\frac{\partial y\left(x_{1}, x_{2}, \ldots, x_{n}\right)}{\partial x_{1}}\right|\right)_{M} \Delta x_{1}+\left(\left|\frac{\partial y\left(x_{1}, x_{2}, \ldots, x_{n}\right)}{\partial x_{2}}\right|\right)_{M} \Delta x_{2}+\ldots \\
& +\left(\left|\frac{\partial y\left(x_{1}, x_{2}, \ldots, x_{n}\right)}{\partial x_{n}}\right|\right)_{M} \Delta x_{n}
\end{aligned}
$$

Here $M \ldots\left(x_{10}, x_{20}, \ldots, x_{n 0}\right)$ is a point in whose surrounding the absolute value is calculated. The error is examined in surrounding of some point always. The higher is position of a point, where the error is investigated, from this point; the lower is accuracy of calculated results.

It is also possible to derive the equation for calculation of the nominal error of the output parameter: 


$$
\begin{aligned}
\Delta_{y} & =\left(\left|\frac{\partial \ln y\left(x_{1}, x_{2}, \ldots, x_{n}\right)}{\partial x_{1}}\right|\right)_{M} \Delta x_{1}+ \\
& +\left(\left|\frac{\partial \ln y\left(x_{1}, x_{2}, \ldots, x_{n}\right)}{\partial x_{2}}\right|\right)_{M} \Delta x_{2}+\ldots \\
& \left.+\left(\mid \frac{\partial \ln y\left(x_{1}, x_{2}, \ldots, x_{n}\right.}{\partial x_{n}}\right) \mid\right)_{M} \Delta x_{n}
\end{aligned}
$$

\section{EXPERIMENTAL}

The model was calculated for the resistance and nonlinearity of adhesive joints. These electrical parameters were studied in dependence on the curing time and the curing temperature. Experiments were carried out at the temperature between $130{ }^{\circ} \mathrm{C}$ and $180{ }^{\circ} \mathrm{C}$, in the time interval 20 to 80 minutes at ambient humidity.

Samples of adhesive joints were prepared using an epoxy resin consisting of bisphenol-A matrix, silver flakes were used as conductive filler. The total percentage of silver in the mixture was $80 \%$ weight.

A four-terminal method was used for the measurement of the electrical resistance and nonlinearity of the current vs. voltage characteristic. The resistance was measured with the use of an LCR meter HP 4284A, nonlinearity with a nonlinearity meter designed and realized at the Department of Electrotechnology.

Results of the measurement of nonlinearity are presented in Tab 1.

Tab. 1 Results of joints nonlinearity measurement

\begin{tabular}{|c|c|c|c|}
\hline \multicolumn{2}{|c|}{$\mathrm{A} 1=130$} & \multicolumn{2}{c|}{$\mathrm{A} 2=180$} \\
\hline $\mathrm{B} 1=20$ & $\mathrm{~B} 2=80$ & $\mathrm{~B} 1=20$ & $\mathrm{~B} 2=80$ \\
\hline$(1)$ & $\mathrm{b}$ & $\mathrm{a}$ & $\mathrm{ab}$ \\
\hline 5.6 & 8.7 & 9.4 & 3.2 \\
\hline 9.4 & 3.2 & 12.3 & 3.5 \\
\hline 7.2 & 8.1 & 7.5 & 1.4 \\
\hline 12.3 & 7.6 & 8.8 & 1.8 \\
\hline 11.6 & 4.6 & 6.2 & 3.9 \\
\hline
\end{tabular}

Results of the measurement of the joints resistance are presented in Tab. 2.
Tab. 2 Results of joints resistances measurement

\begin{tabular}{|c|c|c|c|}
\hline \multicolumn{2}{|c|}{$\mathrm{A} 1=130$} & \multicolumn{2}{c|}{$\mathrm{A} 2=180$} \\
\hline $\mathrm{B} 1=20$ & $\mathrm{~B} 2=80$ & $\mathrm{~B} 1=20$ & $\mathrm{~B} 2=80$ \\
\hline$(1)$ & $\mathrm{B}$ & $\mathrm{a}$ & $\mathrm{ab}$ \\
\hline 32.5 & 20.3 & 12.3 & 13.2 \\
\hline 28.4 & 18.1 & 14.7 & 16.4 \\
\hline 26.2 & 21.4 & 15.1 & 12.9 \\
\hline 37.9 & 19.7 & 11.4 & 24.7 \\
\hline 34.8 & 16.5 & 13.5 & 13.5 \\
\hline
\end{tabular}

Following models were found:

1. Model for joint nonlinearity:

$y=6,82-1,015 x_{1}-2,215 x_{2}-0,825 x_{1} x_{2}$

2. Model for the joint resistance:

$y=20,175-5,405 x_{1}-2,505 x_{2}+3,875 x_{1} x_{2}$

After reverse transformation:

1. Model for joint nonlinearity:

$U_{\text {nelin }}=1,46+0,0144 A+0,0967 B-0,0011 A B$

2. Model for the joint resistance:

$R=68,794-0,4742 A-0,8835 B+0,0052 A B$

Tolerance models:

1. Model for joint nonlinearity:

$$
\begin{aligned}
\Delta U_{\text {nelin }}= & (0,0144+0,0011 B)_{M} \Delta A+(0,0967+ \\
& +0,0011 A)_{M} \Delta B
\end{aligned}
$$

2. Model for the joint resistance:

$$
\begin{aligned}
\Delta R= & (0,4742+0,0052 B)_{M} \Delta A+(0,8835+ \\
& +0,0052 A)_{M} \Delta B,
\end{aligned}
$$

where $A \ldots$ the temperature $\left({ }^{\circ} \mathrm{C}\right), B \ldots$ time of curing (min).

$M(A, B) \ldots$ point of tolerance investigation, $M(155,50)$.

The tolerance models are valid for the interval of the curing temperature $130-180{ }^{\circ} \mathrm{C}$ and the curing time 20-80 minutes. When these models would be used for other interval of the curing parameters, the results would be less accurate. 


\section{DisCUSSION OF RESULTS}

Model, which was found for nonlinearity showed that nonlinearity of the adhesive joints depends, in the interval of curing parameters, which was studied, more on the curing time than on the curing temperature. As for the joint resistance the dependence was similar, but it was not so strong. The interaction of factors $A$ and $B$, expressed as a product $A B$, has the lowest influence on the electrical parameters of the joint, which were tested. Tolerance models are strongly dependent on the mathematical models of the process.

Analysis of the tolerance model for nonlinearity of the current vs. voltage characteristic of adhesive joints shows that the value of the sum in the first parentheses for the middle curing time 50 minutes is approximately 0,07 , whereas the value of the sum in the second parentheses is approximately 0,2 for the middle curing temperature $155^{\circ} \mathrm{C}$. It means, that nonlinearity tolerance depends more on the tolerance of the curing parameter B (the curing time) than on the curing parameter $\mathrm{A}$ (the curing temperature).

Analysis of the tolerance model for the resistance of adhesive joints show that the value of the sum in the first parentheses is approximately 0,72 for the middle curing temperature $155^{\circ} \mathrm{C}$ and the sum in the second parentheses is approximately 1,6 for the middle curing time $50 \mathrm{~min}$. It means, that the tolerance of the adhesive joint resistance depends more on the tolerance of the curing parameter B (the curing time) than on the curing parameter $\mathrm{A}$ (the curing temperature). In this case is this dependence stronger than in the case of joint nonlinearity.

The tolerance models show the tolerances of basic electrical parameters of adhesive joints depend on the values of the curing temperature and the curing time and on the values of their tolerances. The higher are these values, the higher are tolerances. The models are linear, minimum tolerances are for lower specification limits.

\section{CONCLUSIONS}

Tolerance design of the curing process of the joints formed of electrically conductive adhesives was carried out. Tolerances of basic electrical parameters of the joints, the resistance and nonlinearity of the current vs. voltage characteristic, were studied in dependence on tolerances of basic curing parameters, the curing time and temperature.

Mathematical models of the process were calculated and used for calculation of tolerance models. It was found that tolerances of the output parameters grow if the curing temperature and curing time increase and if tolerances of these parameters increase. It was found that tolerances of electrical parameters of adhesive joints depend more on tolerance of the curing time than on tolerance of the curing temperature.

This is valuable information, because curing time can be controlled easier and with higher accuracy than the curing temperature.

Tolerance design is an integral part of a process analysis. Process optimization is impossible without calculation of a mathematical model of a process and without tolerance analysis of this model. This "theoretical" part has to be followed with a practical one, when the conclusions found theoretically are applied on the production line.

This "theoretical" part of process optimization is not too financially demanding and affords significant contribution to the final result.

\section{ACKNOWLEDGMENT}

The authors would like to thank Ministry of education, youth and sports for the financial support (project "Diagnostics of Materials", no. MSM6840770021).

\section{REFERENCES}

[1] ANSI Y 14.5m-1982(R1988) Standard

[2] MONTGOMERY, D. C.: "Introduction to Statistical Quality Control", J. Wiley and Sons, 2001

[3] CONDRA L.W.:"Reliability Improvement with Design of Experiments", Technology and Engineering, 2001

[4] LAMPS, M. F., ECKERT, E. C.: "Improving the suspension design process by integrating multibody system analysis and design of experiments", SAE, 1993

[5] TAGUCHI, G.:"Introduction to Quality Engineering", American Supplier Institute, 1986

[6] CREVELING, C. M., SLUTSKI, J. L., ANTIS, D. Jr.: "Design for Six Sigma in Technology and Product Development”, Prentice Hall PTR, 2003 